\title{
Gambling-Related Distortions and Problem Gambling in Adolescents: A Model to Explain Mechanisms and Develop Interventions
}

\author{
Maria Anna Donati ${ }^{1 *}$, Francesca Chiesi ${ }^{1}$, Adriana Iozzi ${ }^{2}$, Antonella Manfredi ${ }^{3}$, \\ Fabrizio Fagni ${ }^{4}$ and Caterina Primi ${ }^{1}$
}

${ }^{1}$ Department of Neurofarba, University of Florence, Florence, Italy, ${ }^{2}$ UFC SerD Zona 1 Firenze, Azienda USL Toscana Centro, Florence, Italy, ${ }^{3}$ Area Dipendenze, Azienda USL Toscana Centro, Florence, Italy, ${ }^{4}$ UFC SerD Pistoia-Valdinievole, Azienda USL Toscana Centro, Florence, Italy

Although a number of gambling preventive initiatives have been realized with adolescents, many of them have been developed in absence of a clear and explicitly described theoretical model. The present work was aimed to analyze the adequacy of a model to explain gambling behavior referring to gambling-related cognitive distortions (Study 1), and to verify the effectiveness of a preventive intervention developed on the basis of this model (Study 2). Following dual-process theories on cognitive functioning, in Study 1 we tested a model in which mindware gap, i.e., susceptibility to the gambler's

OPEN ACCESS

Edited by:

Xavier Noel,

Université Libre de Bruxelles, Belgium

Reviewed by:

Celine Baeyens,

Université Grenoble Alpes, France

Jeffrey L. Derevensky,

McGill University, Canada

${ }^{*}$ Correspondence:

Maria Anna Donati mariaanna.donati@unifi.it

Specialty section: This article was submitted to

Psychopathology, a section of the journal

Frontiers in Psychology

Received: 21 February 2017 Accepted: 11 December 2017

Published: 05 January 2018

Citation:

Donati MA, Chiesi F, lozzi A, Manfredi A, Fagni $F$ and Primi $C$ (2018) Gambling-Related Distortions and Problem Gambling in Adolescents: A Model to Explain

Mechanisms and Develop Interventions. Front. Psychol. 8:2243. doi: 10.3389/fpsyg.2017.02243 fallacy, and contaminated mindware, i.e., superstitious thinking, were the antecedents of gambling-related cognitive distortions that, in turn, affect gambling frequency and problem gambling. Participants were 306 male adolescents ( $M_{\text {age }}=17.2$ years). A path analysis indicated that cognitive distortions have a mediating role in the relationship that links probabilistic reasoning fallacy and superstitious thinking with problem gambling. Following these findings, in Study 2 we developed a school-based intervention aimed to reduce gambling-related cognitive distortions acting on the above cited mindware problems. A pre- and post-test design - with a 6 months follow-up - was performed with 34 male adolescents ( $M$ age $=16.8$ ), randomly assigned to two groups (Training and No Training), and their baseline equivalence was verified. A Mixed $2 \times 2$ ANOVA attested a significant Time X Group interaction, indicating a significant reduction of the cognitive distortions from pre-test to post-test only in the Training group. The follow-up attested to the stability of the training effects and the reduction of gambling frequency over time. These findings suggest that prevention strategies should address mindware problems, which can be considered as predictors of gambling-related cognitive distortions.

Keywords: gambling, prevention, adolescents, gambling-related cognitive distortions, mindware, gambler's fallacy, superstitious thinking

\section{INTRODUCTION}

Despite the restrictions to gamble for youth, prevalence studies report that a large number of adolescents are involved in gambling activities and that they are at higher risk for developing gambling problems compared to adults (see Blinn-Pike et al., 2010; Volberg et al., 2010, for reviews). There are alarming data as initiation of gambling at an early age is associated with a 
higher risk of more severe gambling problems in adulthood (Johansson et al., 2009; Granero et al., 2014). Due to the potential negative consequences derived from gambling, prevention of problem gambling among adolescents has increasingly become an important area of concern in research and practice. For this reason, several educational initiatives have been realized (see Ladouceur et al., 2013; St-Pierre et al., 2015; Keen et al., 2016, for reviews). However, many of them have been developed in absence of an explicitly described theoretical model (Ladouceur et al., 2013; St-Pierre et al., 2015) and, even when a theoretical model has been proposed, it was adapted from other addictions' prevention approaches or it is often unclear how the theory was used in the program development (St-Pierre et al., 2015).

Following this premise, the goal of the present work was to fill this gap through two studies. In Study 1, we aimed to test a theoretically grounded model to explain gambling frequency and problem gambling referring to gambling-related cognitive distortions, i.e., a wide array of mistaken beliefs and perceptions about gambling (Raylu and Oei, 2004; Johansson et al., 2009). Then, in Study 2, we aimed to develop and verify the effectiveness of a preventive intervention focused on gamblingrelated cognitive distortions based on the model tested in Study 1.

\section{STUDY 1}

Referring to research with adults, dual-process theories on cognitive functioning have been used to explain cognitive failure that leads to persistent gambling behavior (Toplak et al., 2007). These theories distinguish between autonomous sets of systems (rapid, automatic, parallel, and heuristic) and analytic cognitive processes (slow, under control, serial, and rule-based) (see Stanovich, 2004, for a review). Toplak et al. (2007) used this model to explain how people tend to be irrational while gambling. Particularly, they considered problems regarding mindware (Perkins, 1995), defined as the rules, procedures, and strategies derived from past learning experiences and available for explicit retrieval. The authors stated that mindware problems can arise when there is a mindware gap or in the case of contaminated mindware. Specifically, there is a mindware gap when the appropriate rules, procedures, and strategies are lacking, while a contaminated mindware verifies when the employed mindware is not helpful in the specific situation. Referring to gambling, to operationalize the mindware gap, they referred to probabilistic reasoning ability in a variety of heuristic and bias problems and they proposed the disposition to believe in paranormal events, superstition, and luck to operationalize the contaminated mindware.

On one hand, a mindware gap in probabilistic reasoning, intended as the ability to draw conclusions about the likelihood of events based on available information or personal knowledge or beliefs, could have an important role in gambling. Indeed, it has been suggested that misunderstanding of probability can lead to irrational thoughts and behaviors related to gambling, such as chasing or obtaining false contingencies (Raylu and Oei, 2004). As reviewed by Goodie and Fortune (2013), misrepresentations about the chance of winning can derive from the representativeness heuristic, i.e., a tendency for people to base their judgment of the probability of a particular event on how much it represents the essential features of the parent population or of its generating process (Kahneman et al., 1982), and associated biases. For instance, one of the most documented biases related to gambling is the gambler's fallacy, which occurs when individuals believe that even short strings of random events must correspond with their perception of what constitutes randomness, leading to beliefs that particular outcomes are "due" (Tversky and Kahneman, 1971).

On the other hand, a contaminated mindware as superstitious thinking, i.e., the propensity of having beliefs based on perceiving biased casual relationships between unrelated events (Ninness and Ninness, 1998), can be related to distortions about gambling. Superstition, which appears during childhood and adolescence (Chiesi et al., 2010), is a thinking disposition that can affect reasoning regardless of cognitive abilities (Sá et al., 2005; West et al., 2008). Research and practice with adult pathological gamblers have shown that they have behavioral superstitions in which they associate certain habits with positive gambling results, cognitive superstitions in which they associate specific thought processes with winning, or talismanic superstitions in which they associate good luck charms with winning (Toneatto, 1999).

Taken together, mindware gap and contaminated mindware, as defined inside the above described dual-process framework, can provide an explanation for the mechanisms under which gambling-related cognitive distortions arise. These distortions, e.g., mistaken perceptions of the role of personal ability in gambling, misrepresentations of the chances of winning, false beliefs about the possibility to control or predict gambling outcomes, are deemed important risk factors for pathological gambling in both adults and adolescents. Indeed, high levels of cognitive distortions have been found to be associated with high levels of gambling frequency and to play an important role in the development of problem gambling in adults (Raylu and Oei, 2004; Arcan and Karanci, 2015; see Fortune and Goodie, 2012, for a review). Consistently, cognitive distortions related to gambling predict the frequency of gambling (Donati et al., 2015) and are strong predictors of problem gambling among adolescents (e.g., Taylor et al., 2014; Cosenza and Nigro, 2015; Donati et al., 2015).

Following these premises, we aimed to test a model in which susceptibility to the gambler's fallacy (mindware gap) and superstitious thinking (contaminated mindware) were associated with gambling frequency and problem gambling through gambling-related cognitive distortions. Indeed, given the importance of cognitive distortions in relation to gambling behavior, it becomes relevant to investigate their possible antecedents in young people. To the best of our knowledge, there are few studies on this topic and, in particular, there is a lack of studies attesting empirically the relationship between probabilistic reasoning and superstition to gambling-related cognitive distortions among adolescents.

We hypothesized that higher susceptibility to the gambler's fallacy and higher superstitious thinking would be related to higher gambling-related erroneous cognitions. Moreover, since both susceptibility to the gambler's fallacy and superstitious thinking have been found to be related to gambling behavior 
among adolescents (e.g., Skoukaskas and Satkeviciute, 2007; Delfabbro et al., 2009; Chiu and Storm, 2010; Donati et al., 2013), we predicted that cognitive distortions related to gambling would mediate the relationship between susceptibility to the gambler's fallacy and superstitious thinking with gambling frequency and problem gambling. Furthermore, as the frequency of gambling has been found to be linked to the number of problem gambling symptoms (Chiu and Storm, 2010; Derevensky et al., 2010), we predicted that gambling-related cognitive distortions would affect problem gambling also indirectly through gambling frequency. Finally, as probabilistic reasoning biases have been found to be related to superstition (e.g., Kokis et al., 2002; Chiesi et al., 2010), we hypothesized a positive correlation between susceptibility to the gambler's fallacy and superstitious thinking.

\section{Methods}

\section{Participants}

Participants included 306 male adolescents $\left(M_{\text {age }}=17.2\right.$ years, $S D=1.5$, range: 14-24) who attended high school in Italy (Tuscany). In line with some studies (e.g., Vitaro et al., 2004; Ricijas et al., 2016), we recruited only boys. Indeed, despite the expansion of the gambling industry has modified the maledominated gambling culture (Dowling, 2013), gender differences in gambling behavior have been reported, indicating that boys are more likely than girls to gamble and to report gambling problems (see Splevins et al., 2010; Calado et al., 2016, for reviews). Written informed assent was provided by students and by the parents if the student was a minor.

\section{Measures and Procedure}

To measure susceptibility to the gambler's fallacy, the Gambler's Fallacy Task (GFT, Primi and Chiesi, 2011) was used. It consists of a marble bag game in which participants were asked which outcome was more likely at the next draw after a sequence of five equal outcomes (five blue or five green marbles). In more detail, the task was composed of three different trials in which the proportion of Blue and Green marbles in the bag varied (first trial: $15 \mathrm{~B}$ and $15 \mathrm{G}$; second trial: $10 \mathrm{~B}$ and $20 \mathrm{G}$; third trial: $25 B$ and $5 G$ ). In total, each participant answered six questions. Summing fallacious answers, we computed a gambler's fallacy score ranging from 0 to 6 , with higher scores corresponding to higher susceptibility to the gambler's fallacy.

To measure superstitious thinking, the Superstitious Thinking Scale (STS, Kokis et al., 2002; Italian version: Chiesi et al., 2010) was used. It is composed of eight Likert-type items using a 5-point scale ranging from totally false to totally true, yielding a maximum score of 40 . Higher scores represent high levels of superstitious thinking. An example of an item is "The number 13 is unlucky". Coefficient alpha for the current sample was satisfactory $(\alpha=0.77)$.

The Gambling Related Cognitions Scale (GRCS; Raylu and Oei, 2004; Italian version: Iliceto et al., 2015) is a self-report scale to assess gambling-related cognitions. It contains twentythree Likert-type items (using a 7-point scale ranging from strongly disagree to strongly agree) related to five biases regarding gambling measured by the following subscales: Gambling Expectancies (4 items; e.g., "Having a gamble helps reduce tension and stress"), Illusion of Control (4 items; e.g., "Specific numbers and colors can help increase my chances of winning"), Predictive Control (6 items; e.g., "When I have a win once, I will definitely win again"), Inability to Stop Gambling (5 items; e.g., "It is difficult to stop gambling as I am so out of control"), and Interpretative Bias (4 items; e.g., "Relating my losses to bad luck and bad circumstances makes me continue gambling"). The scale was previously found to have adequate validity and reliability among adolescents (e.g., Taylor et al., 2014; Donati et al., 2015). The coefficient alpha for the current sample was satisfactory $(\alpha=0.89)$. The GRCS subscale scores as well as the GRCS total score, obtained by summing the score for each item, were calculated. However, following the suggestion that only the total score for the GRCS should be used with adolescents (Taylor et al., 2014), the total score was used in the path model.

Gambling behavior was measured through the South Oaks Gambling Screen-Revised for Adolescents (SOGS-RA; Winters et al., 1993; Italian version: Colasante et al., 2014). This is one of the most widely instrument to measure problem gambling with adolescents (see Edgren et al., 2016, for a review), and its effectiveness has been attested by applying Item Response Theory (Chiesi et al., 2013). The scale is composed of two sections using the last year gambling behavior. In the first one, participants were asked to indicate the frequency of gambling (Never $=0$, Less Than Monthly = 1, Monthly = 2, Weekly = 3, and Daily = 4) among a list of eleven gambling activities including: Playing cards for money, coin tosses for money, bets on games of personal skill, bets on sports teams, bets on horse or dog races, bingo, dice games for money, slot machines, scratch-cards, lotteries, and on-line games. Considering responses to this section, participants can be classified into non-gamblers (no gambling behavior) and gamblers (gambling on at least one activity) (Welte et al., 2009). Moreover, among gamblers, non-regular gamblers (i.e., those who participated from less than monthly to less than weekly in at least one gambling activity) and regular gamblers (i.e., those who participated weekly or daily in at least one gambling activity) can be identified (Winters et al., 1993). Finally, a total score of gambling frequency (range: $0-44$ ) can be obtained by summing the responses for each gambling activity (Wickwire et al., 2007). The second section consists of 12 items related to the Diagnostic and Statistical Manual of Mental Disorders (III edition revised) criteria for pathological gambling (American Psychiatric Association, 1987). An example is: "In the past 12 months, how often have you gone back another day to try to win back money that you lost?". All items require dichotomous answers (i.e., yes or no) except the first item, which has a 4-point response scale (never, some of the time, most of the time, every time), and it is dichotomized (i.e., never/some of the time or most of the time/every time) in the scoring phase. A single composite score was computed summing the responses for each item of the second section. The total SOGS-RA score, indicative of the number of problem gambling symptoms, was used as dependent variable (range: $0-12$ ), in line with previous studies (e.g., Wickwire et al., 2007, 2010). Finally, according to the narrow criterion (Winters et al., 1995), different categories of gamblers were identified: Non-problem gamblers (i.e., SOGSRA scores from 0 to 1 ), at-risk gamblers (i.e., SOGS-RA scores 
TABLE 1 | Descriptive statistics for gambler's fallacy, superstitious thinking, gambling-related cognitive distortions - the GRCS total score and the subscale scores - and problem gambling for non-regular gamblers $(n=86)$ and Regular gamblers $(n=168)$.

\begin{tabular}{|c|c|c|c|c|c|}
\hline \multirow[b]{2}{*}{ Dependent variable } & \multicolumn{2}{|c|}{$\begin{array}{c}\text { Type of gamblers based upon } \\
\text { gambling frequency }\end{array}$} & \multirow[b]{2}{*}{$t$ (df) } & \multirow[b]{2}{*}{$p$} & \multirow[b]{2}{*}{ Cohen's $d$} \\
\hline & $\begin{array}{c}\text { Non-regular } \\
\text { gamblers } M(S D)\end{array}$ & $\begin{array}{c}\text { Regular } \\
\text { gamblers } M(S D)\end{array}$ & & & \\
\hline Gambler's fallacy & $4.56(1.73)$ & $5.19(1.44)$ & $-2.74(252)$ & $<0.01$ & 0.40 \\
\hline Superstitious thinking & $18.49(6.25)$ & $21.36(7.10)$ & $-3.30(252)$ & $<0.01$ & 0.43 \\
\hline Gambling related cognitive distortions - Total score & $35.68(12.31)$ & $48.66(20.36)$ & $-6.32(252)$ & $<0.001$ & 0.77 \\
\hline Gambling Expectancies & $5.39(2.08)$ & $8.55(4.64)$ & $-7.49(252)$ & $<0.001$ & 0.88 \\
\hline Illusion of Control & $5.35(2.50)$ & 7.07 (3.99) & $-4.20(252)$ & $<0.001$ & 0.70 \\
\hline Predictive Control & $11.65(5.67)$ & $14.42(6.36)$ & $-3.53(252)$ & $<0.001$ & 0.46 \\
\hline Inability to Stop Gambling & $6.54(2.68)$ & $8.46(2.57)$ & $-3.87(252)$ & $<0.001$ & 0.73 \\
\hline Interpretative Bias & $6.75(3.75)$ & $10.16(5.44)$ & $-5.86(252)$ & $<0.001$ & 0.73 \\
\hline Problem gambling & $0.60(1.06)$ & $1.81(2.07)$ & $-6.20(252)$ & $<0.001$ & 0.74 \\
\hline
\end{tabular}

from 2 to 3), and problem gamblers (i.e., SOGS-RA scores of 4 or more).

The above-described scales were administered in the classrooms and students were required to work individually. Teachers were not present during the administration of the scales, which required approximately $40 \mathrm{~min}$.

\section{Results}

Results showed that $16 \%$ of the participants had never gambled. Then, we performed the analyses on adolescent gamblers, i.e., the 254 respondents who affirmed having gambled at least once during the last year. Among them, 66\% were non-regular gamblers $(n=86)$, and $34 \%$ were regular gamblers $(n=168)$. The most common activities were scratch-cards (74\%), sport bets (62\%), and cards for money (47\%), while the least engaged in activities were dice games for money (7\%), bets on coin tosses $(8 \%)$, and bets on horse or dog races (9\%). Considering the score of the second section of the SOGS-RA, 75\% $(n=190)$ of the respondents were non-problem gamblers, $19 \%(n=48)$ at-risk gamblers, and 6\% $(n=16)$ problem gamblers. Descriptive statistics of GFT, STS, GRCS, and the SOGS-RA based upon gambling frequency are displayed in Table 1, while descriptive statistics of the scales for the entire sample are reported in Table 2.

As reported in Table 1, results showed that regular gamblers were more susceptible to the gambler's fallacy, had higher levels of superstitious thinking and gambling related cognitive distortions, and reported more problem gambling symptoms than nonregular gamblers.

Then, we computed Pearson correlations to investigate the relationships among susceptibility to the gambler's fallacy, superstitious thinking, gambling-related cognitive distortions the GRCS total score and the subscale scores-, gambling frequency, and problem gambling.

As shown in Table 2, gambling-related cognitive distortions were significantly and positively correlated both with susceptibility to the gambler's fallacy and superstitious thinking. In detail, with the exception of Interpretative Bias, all the five cognitive distortions were related to susceptibility to the gambler's fallacy and superstitious thinking, especially Illusion of Control, which shows, respectively, moderate and high correlations with the two variables. In addition, gambling-related cognitive distortions were significantly and positively correlated both with gambling frequency and problem gambling. Looking at GRCS subscales correlations, results indicated moderate and high correlations, with the highest between gambling frequency and Gambling Expectancies, while, Inability to Stop Gambling showed the highest Pearson coefficient value in the association with problem gambling. The results also showed that susceptibility to the gambler's fallacy was significantly and positively correlated with superstitious thinking, and both these variables were significantly and positively correlated with gambling frequency. The correlations between problem gambling were not significant. Finally, gambling frequency resulted to be significantly and positively correlated with problem gambling.

To investigate our hypothesis on the mechanisms underlying the relationships among these variables, we conducted a path analysis with AMOS using maximum likelihood estimation. The model included susceptibility to the gambler's fallacy and superstitious thinking as gambling-related cognitions' antecedents, and gambling-related cognitive distortions as antecedents of gambling frequency and problem gambling, which was directly affected by gambling frequency (Figure 1). Several goodness-of-fit indices were used to test the adequacy of the model: The Comparative Fit Index (CFI; Bentler, 1990), the Tuker-Lewis index (TLI; Tucker and Lewis, 1973), and the Root Mean Square Error of Approximation (RMSEA; Steiger and Lind, 1980). CFI and TLI values equal to.90 or greater (Tucker and Lewis, 1973; Bentler, 1990) and RMSEA values of.08 or below (Steiger and Lind, 1980) were considered as indices of adequate fit.

The hypothesized model showed a good fit to the data $(\mathrm{CFI}=0.98$, TLI $=0.94$, RMSEA $=0.07)$. All coefficients were statistically significant and in the expected directions. Specifically, results revealed that susceptibility to the gambler's fallacy and superstitious thinking had significant direct positive effects on gambling-related cognitive distortions. Gambling-related cognitive distortions were directly and 


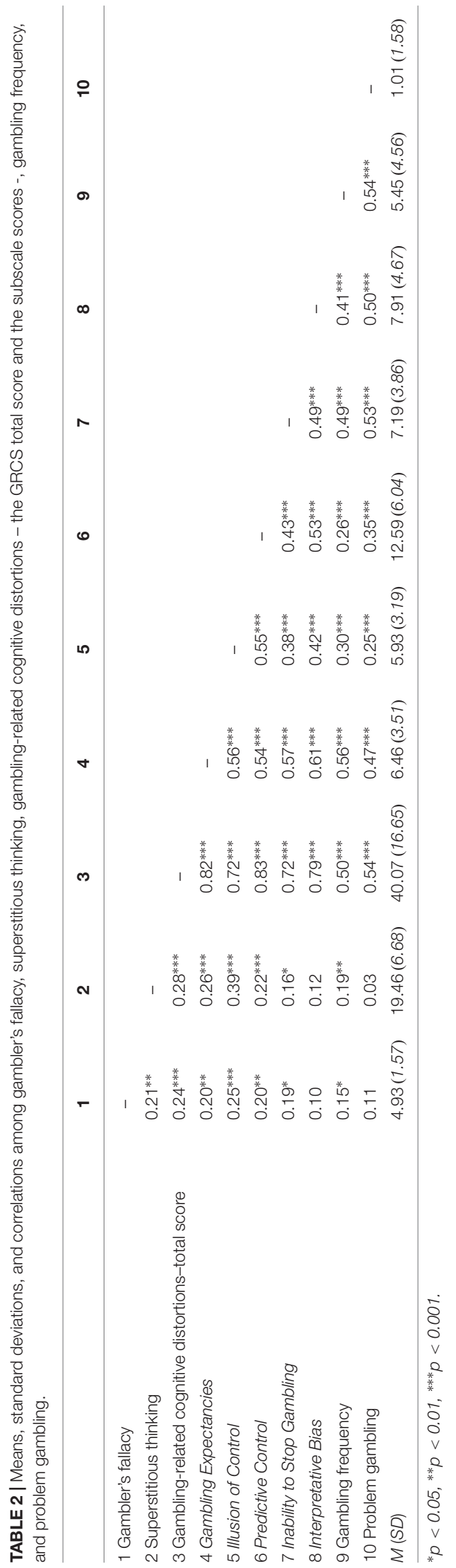

positively related to gambling frequency as well as problem gambling, and gambling frequency had a direct positive effect on problem gambling. Moreover, both susceptibility to the gambler's fallacy and superstitious thinking had significant indirect effects on gambling frequency, respectively $0.09(p<0.01)$ and $0.12(p<0.01)$, and on problem gambling, respectively $0.10(p<0.01)$ and $0.13(p<0.01)$. A significant indirect effect of $0.18(p<0.01)$ was also found between gambling-related cognitions and problem gambling Finally, a significant positive covariation was found between susceptibility to the gambler's fallacy and superstitious thinking.

\section{Discussion}

The aim of this study was to test the adequacy of a model explaining the relationship between susceptibility to the gambler's fallacy, superstitious thinking, gambling-related cognitive distortions, and gambling behavior among adolescents. In line with the predictions, findings revealed that the tendency to commit the gambler's fallacy and to be superstitious affects distorted cognitions about gambling. More specifically, higher susceptibility to commit the gambler's fallacy and higher superstitious thinking were related to greater levels of gamblingrelated cognitive distortions. Moreover, our model showed that cognitive distortions on gambling mediate the relationship between susceptibility to the gambler's fallacy and superstitious thinking with gambling behavior.

From a theoretical point of view, our findings confirm that there may be a cognitive-psychological mechanism through which faulty beliefs about gambling develop. In particular, results are consistent with Toplak et al.'s (2007) suggestions about the existence of two cognitive processes that affect problematic gambling behavior in adults-following a dual-process perspective on cognitive functioning (Stanovich, 2004)- i.e., difficulties in dealing with probability (mindware gap) and belief in superstition and luck (contaminated mindware).

Compared to previous research with adolescents, our study expands the current knowledge by suggesting that gambler's fallacy and superstitious thinking are related to gambling-related distortions in adolescence. Furthermore, this study suggests that the relationship between gambler's fallacy and problem gambling (e.g., Skoukaskas and Satkeviciute, 2007; Delfabbro et al., 2009; Donati et al., 2013), and the association of superstitious thinking with problem gambling (e.g., Chiu and Storm, 2010; Donati et al., 2013) can be explained by taking into account the mediating role of gambling-related cognitive distortions. In other words, adolescents more prone to mistaken perceptions of probability and with the tendency to adhere to superstitious beliefs are susceptible to cognitive distortions related to gambling. As such, they are particularly at risk since they have a greater likelihood of gambling with high frequency and developing gambling problems.

Although this model has been verified with a relatively small number of adolescent male gamblers, practical implications can be derived from the study. Indeed, our model can represent a theoretical based framework from which developing theorydriven interventions oriented to youth. Specifically, focusing on 


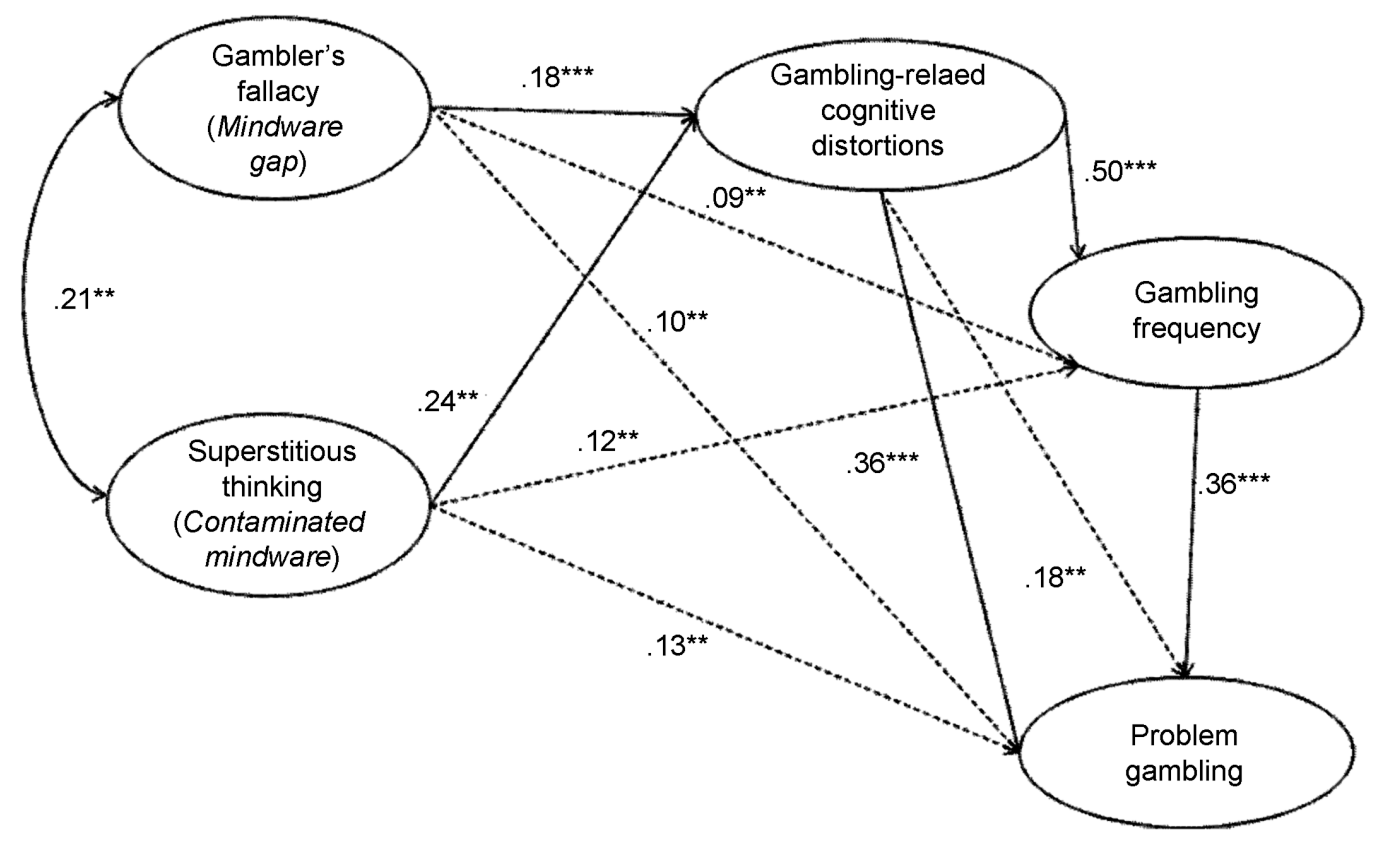

FIGURE 1 | Model of problem gambling with standardized parameters (significant path coefficient $* * *$ at the 0.001 level, ** at the 0.01 level). Dotted lines represent indirect effects, while continuous lines indicate direct effects.

these findings, a program aimed to modify gambling-related cognitive distortions can be developed. This was the aim of Study 2.

\section{STUDY 2}

Among the guidelines published by the Society for Prevention Research for the development of effective preventive interventions (Flay et al., 2005), there is the desirable standard that a clear theory of causal mechanism of the change promoted by the intervention should be stated. In particular, it would be important that the preventive program would be informed by theory and prior empirical analyses on antecedents and predictors of outcomes. Indeed, the systematic reviews conducted on the preventive interventions developed with adolescents in the school setting (Ladouceur et al., 2013; St-Pierre et al., 2015; Keen et al., 2016) agree in recognizing that many of the existing prevention programs have been developed in absence of a clear theoretical framework describing the expected causal mechanisms by which the programs would exert their effect.

Following this premise, once tested the adequacy of our model on gambling in Study 1, the goal of Study 2 was to develop and verify the effectiveness of a universal preventive intervention addressed to general samples of youth, regardless of risk or gambling status (Ladouceur et al., 2013; Keen et al., 2016) aimed to reduce gambling-related cognitive distortions by acting on probabilistic reasoning errors (mindware gap) and superstitious thinking (contaminated mindware). Additionally, moving from the theoretical guideline for which the main purpose of any prevention program should be to reduce the incidence of the potential problem (Ladouceur et al., 2013), we aimed to obtain behavioral changes related to gambling frequency, which is an antecedent of problem gambling (Chiu and Storm, 2010; Derevensky et al., 2010). Finally, due the "preventive" nature of the current intervention, reducing the incidence of problem gambling was outside our goals. In sum, following St-Pierre et al.'s (2015) classification framework, we developed a "gamblingspecific psychoeducational and skills training prevention program" to reduce the erroneous cognitions on gambling acting on gambling-related knowledge, beliefs, attitudes, and skills as well as the awareness about the nature of gambling, knowing that all these factors may impact on adolescents' gambling habits.

In evaluating the effectiveness of the proposed intervention, we also wanted to take into account some relevant methodological issues. First of all, although a short-term change of gambling-erroneous cognitions have been obtained in several of these preventive initiatives (e.g., Ferland et al., 2002, 2005; Capitanucci et al., 2010; Williams et al., 2010; Donati et al., 2014; Huic et al., 2017), only few of them verified the stability of these effects over time (Gaboury and Ladouceur, 1993; Capitanucci et al., 2010; Donati et al., 2014). Thus, to provide evidence of the strength and stability of the change in the current intervention program, we assessed the short-term and long-term effects on gambling-related cognitions and also the long-term effects on gambling behavior. Secondly, we employed scales (i.e., SOGS-RA, GRCS, GFT, and STS) that were previously analyzed for their psychometric properties (see Study 1 for a detailed description). Indeed, the majority of the gambling intervention programs have not used psychometrically good measurement instruments to assess the variables of interest despite their obvious necessity (Ladouceur et al., 2013). Finally, 
we employed an experimental design in which we verified the baseline equivalence of the experimental group and control group for the targeted variables of our intervention. Even in this case, with few exceptions (Williams et al., 2010; Donati et al., 2014), the baseline equivalence between the experimental and control groups has not been tested in past studies.

\section{Methods}

\section{Participants}

Participants were 34 male high school students $\left(M_{\text {age }}=16.80\right.$, $S D=1.04$, range: $15-19)$ enrolled in a public high school in Tuscany (Italy). From the available schools in the area, one school was randomly selected. Subsequently, the school's principal was contacted, apprised of the issue of adolescent problem gambling to generate support for the research, and he was presented with the project. Once the school agreed to participate, the detailed study protocol was approved by the institutional review board of the school. Written informed consent was requested from students (or their parents, if they were minors), assuring them that the data would be handled confidentially. The research was conducted during school time and all students invited to participate agreed to do so. We chose a specific sample as it seems pertinent to deliver interventions to small groups of students that are homogenous in terms of risk factors, gambling habits, gender, and age (Ladouceur et al., 2013).

\section{Measures}

In line with Study 1, participants were administered the GFT, the STS, the GRCS, and the SOGS-RA (see Study 1 for description and scoring).

\section{Procedure and Design}

To evaluate changes in the dimensions considered in the study over time as a function of treatment condition, an experimental design was conducted with two groups (Training vs. No Training) and three measurements (pre-test, post-test, and follow-up sessions). Classes were randomly assigned to the Training and No Training conditions. The Training group consisted of 16 students $\left(M_{\text {age }}=16.99, S D=1.20\right)$ and the No Training group consisted of 18 students $\left(M_{\text {age }}=16.63\right.$, $S D=0.89)$. For the Training group, participation involved filling out the above described scales before the intervention (pre-test), receiving training activities, filling out the GRCS after intervention (post-test), and then compiling the GRCS and the SOGS-RA six months (school break over the summer occurred during this interval) after the intervention has ended (follow-up). The pre-test, post-test, and follow-up questionnaires were administered also to the No Training group. Nevertheless, while the Training group received the intervention, the No Training group continued with usual school activity.

In the pre-test, post-test, and follow-up sessions, the scales were administered within the classrooms, and students were required to work individually. Teachers were not present during the administration of the scales. Administration of the instruments required approximately $40 \mathrm{~min}$ for the pre-test session, $15 \mathrm{~min}$ for the post-test session, and $25 \mathrm{~min}$ for the follow-up session. The Training group attended the intervention approximately 2 weeks after the pre-test, and the post-test was administrated 1 week after the end of the intervention and 5 weeks after pre-test data were collected. Few days after the follow-up session, a final meeting took place during which all the participants were given a feedback about the research and thanked for their participation.

\section{The Intervention}

Our intervention activities were based upon the model tested in Study 1. In detail, as cognitive distortions on gambling are affected by problems regarding mindware gap, i.e., probabilistic reasoning errors, and contaminated mindware, i.e., superstitious thinking, we wanted to implement activities in which adolescents could reinforce their ability to recognize biases in reasoning with randomness and could reflect about the irrationality of superstitions. Specifically, as for the mindware gap, activities focused on: Randomness with a series of coin tosses, independent random events employing a 40 cards desk, independence with equally likely and non-equally likely events represented with different colored paper sheets, gambler's fallacy in no-gambling and gambling contexts, and probabilistic reasoning in fictitious gambling situations. Regarding the contaminated mindware, participants were told about the superstition meaning and the lack of cause-effect relationship between a supposed event bringing bad or good luck and the supposed positive or negative event occurred referring to common superstitions. Then, referring more specifically to the relationship between superstition and gambling, several examples were presented about susceptibility to superstitious conditioning in gambling activities and the absence of a causal relationship between superstitious thoughts (e.g., the belief in lucky numbers) and gambling outcomes.

Concerning the training techniques, we integrated a mixed set of techniques including activities with random events generators, Power-Point presentations, and collective discussions. As for the methodology, each didactic unit included exercises in which students had to apply the learned ability/concept, and then they had to use the learned ability referring to fictitious gambling situations. In that way, training activities were aimed to promote the generalization of the proposed contents in real-life contexts. Concerning the procedure, each activity was implemented using a specific sequence: Initial instructions by the trainer, running the activity by the students, interactive discussion and synthesis of the contents, delivery of summary sheets to the students.

The intervention included two didactic units implemented in class, during the normal school time conducted by a developmental psychologist expert in the field of adolescent gambling research with a couple of operators belonging to the addiction unit of the socio-territorial service. Teachers were not present during the administration of the training program. Each didactic unit lasted about $2 \mathrm{~h}$ and were presented in a 2 week period (one per week).

\section{Results}

Results showed that $85 \%$ of the participants $(n=29)$ affirmed having gambled at least once during the last year. 
Among them, 76\% were non-regular gamblers, and $24 \%$ were regular gamblers. The most common activities were scratchtickets (62\%), sport bets (41\%), and cards for money (23\%). Considering the score of the second section of the SOGS-RA, $81 \%(n=23)$ of the respondents were non-problem gamblers, $12 \%(n=4)$ at-risk gamblers, and $6 \%(n=2)$ problem gamblers.

Preliminarily, we tested the baseline equivalence of the Training and No Training groups for age and the targeted variables of our intervention. No significant differences were found between the two groups concerning age $(p=0.316)$, susceptibility to the gambler's fallacy $(p=0.111)$, and superstitious thinking $(p=0.661)$. Then, we analyzed the short-term efficacy of the intervention conducting a Mixed $2 \times 2$ ANOVA with Time (pre- and post-test) as within factor, Group (Training and No Training) as between factor, and gambling-related cognitive distortions as dependent variable.

A significant Time $\times$ Group interaction was found $\left[F(1,32)=4.25, p<0.05, \eta_{\mathrm{p}}^{2}=0.117\right]$. Post hoc $t$-tests showed the interaction effects to be due to significant changes from pre-test to post-test in the Training group but not in the No Training group. Specifically, in the Training group there was a significant reduction of gambling-related cognitive distortions $[t(15)=2.78, p<0.05$, Cohen's $d=0.69)$ from pre-test $(M=37.31, S D=17.53)$ to post-test $(M=25.88, S D=5.25)$, while no significant changes occurred in the No Training group $[t(17)=-0.61, p=0.552]$ from pre-test $(M=37.61$, $S D=10.95)$ to post-test $(M=41.11, S D=26.43)$. Moreover, the two groups resulted to be significantly different for gamblingrelated cognitive distortions at the post-test $[t(32)=-2.26$, $p<0.05$, Cohen's $d=0.80$ ]. The Training group resulted to have lower levels of erroneous cognitions about gambling compared with the No Training group, while at the pre-test they have an equivalent level (Figure 2).

To verify the stability of the short-term effects over time, for the Training participants, we compared post-test and follow-up scores of gambling-related cognitive distortions. In detail, using paired $t$-tests, we compared post-test scores with the follow-up ones. Results showed no significant differences $[t(15)=-0.29$, $p=0.780$ ] suggesting the permanence of the intervention effects over time for gambling-related cognitive distortions from post-test $(M=25.88, S D=5.25)$ to follow-up $(M=26.31$, $S D=6.66)$.

Subsequently, to verify whether the intervention had a decrementing effect on adolescent self-reported gambling behavior, a Mixed $2 \times 2$ ANOVA with Time (pre- and follow-up) as within factor, Group (Training and No Training) as between factor, and gambling frequency as dependent variable, was conducted. A non-significant Time $\times$ Group interaction was found $[F(1,32)=1.70, p=0.201]$. Nonetheless, since the sample size was small and important effect might be non-significant (i.e., Type II errors might be made), we looked at the effect size $\left(\eta_{\mathrm{p}}^{2}=0.05\right)$, which suggested that a small effect was obtained. As such, post hoc $t$-tests showed significant changes from pre- to follow-up in the Training group but not in the No Training group. Specifically, in the Training group there was a significant and medium size change of gambling frequency $[t(15)=2.95, p<0.05$, Cohen's $d=0.73$, suggesting a reduction of gambling frequency from pre-test $(M=3.69, S D=4.96)$ to follow-up $(M=1.50$, $S D=2.53)$. On the contrary, no significant changes occurred in the No Training group $[t(17)=0.04, p=0.969]$ from pre-test $(M=3.94, S D=4.45)$ to follow-up $(M=3.89$, $S D=5.22)$.

\section{Discussion}

Following the results of Study 1, the aim of the present study was to develop and evaluate a preventive intervention which would be able to modify erroneous cognitions about gambling by acting on probabilistic reasoning biases (mindware gap) and superstitious thinking (contaminated mindware). Findings showed that the intervention produced the hypothesized effects in the short-term as participants who attended the training program reduced their gambling-related cognitive distortions, while the participants who did not follow the training program

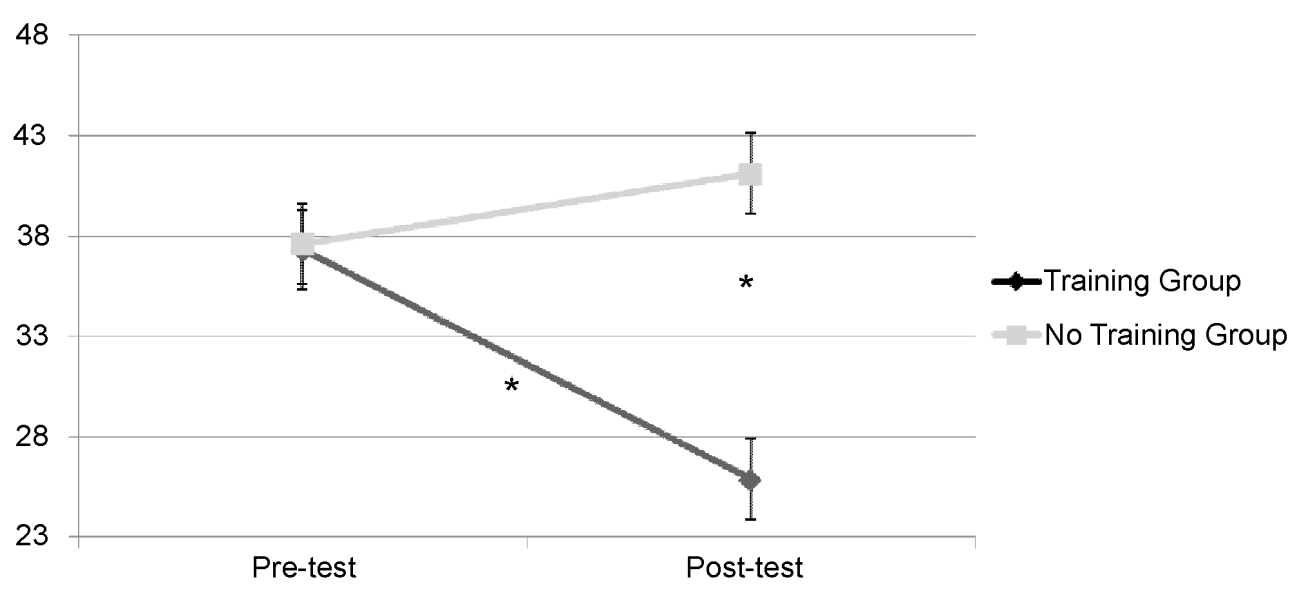

FIGURE 2 | Gambling-related cognitive distortions by Time (pre-test and post-test) and group (Training and No Training). ${ }^{*} p<0.05$. 
did not show a significant change from pre-test to posttest. This finding is of particular importance since research has generally provided evidence of a resistance to change for probabilistic reasoning biases (for a summary of the literature, see e.g., Gilovich et al., 2002; specifically, for adolescents, see Klaczynski, 2004). Additionally, the above described short-term results for gambling erroneous distortions were found to be stable after 6 months by the end of the intervention (i.e., the post-test mean scores did not differ significantly from the follow-up), indicating a substantial persistence of the effects over a period of six months for participants attending the training program.

Concerning the effects on gambling behavior, whereas some previous studies reported no behavioral changes despite improvements in knowledge and the reduction of cognitive errors (Gaboury and Ladouceur, 1993; Ferland et al., 2005; Turner et al., 2008; Huic et al., 2017), some changes were produced in gambling behavior. Specifically, in line with previous studies (Williams, 2002; Donati et al., 2014) we observed that only adolescents who attended the training program reduce their gambling frequency from pre-test to follow-up.

Finally, the methodological strengths of the current study, i.e., having tested short-and long-term effect of the intervention, having used effective instruments to measure the variables of interest, and having tested the baseline equivalence of the experimental and control group, attest to the worth and utility of the proposed intervention.

In sum, the current study provided evidence about the effectiveness of an intervention based upon an evidence-based theoretical model referring to the dual-process theoretical framework.

\section{GENERAL DISCUSSION}

The systematic reviews (Ladouceur et al., 2013; St-Pierre et al., 2015; Keen et al., 2016) conducted on the preventive interventions developed with adolescents in the school setting agree in recognizing that many of the existing prevention programs have been developed in absence of a clear theoretical framework describing the expected causal mechanisms by which the programs would exert their effect. Overcoming the limitations of the previous studies, this work proposed and tested the effectiveness of a gambling preventive intervention with adolescents after having previously verified the adequacy of a theoretical model explaining adolescent gambling involvement. With respect to the application of dual-process theories on cognitive functioning in the prediction of gambling behavior (Toplak et al., 2007), our model proposed that susceptibility to the gambler's fallacy and superstitious thinking (respectively, mindware gap and contaminated mindware, according to the dual-process theory) were the predictor variables of gambling-related distorted cognitions in adolescence, while gambling frequency and problem gambling were the outcome variables. This study supports the results suggested by Clark (2010) that the high propensity for individuals commit mistakes in reasoning and judgment makes them particularly vulnerable to adhere and maintain cognitive distortions related to gambling. With specific reference to the practical implications of the model, our results provided a theoretically grounded model useful not only to explain gambling-related cognitions but also to develop interventions to modify them.

More broadly speaking, this work showed that dual-process theory of cognition (see Evans and Stanovich, 2013; for a review) can be used as conceptual framework to explain and prevent gambling behavior in adolescence. There have been only few attempts to apply it to adolescents, with few exceptions (see Klaczynski, 2004, for the employment of this theory to explain adolescent social and cognitive development), and little research has been conducted in order to investigate its application in the field of adolescent health behavior. Nevertheless, it has been suggested the utility of dual-process theories in explaining and predicting many types of health behaviors in adolescence as they involve both analytic and heuristics processing (Gibbons et al., 2009). Thus, the present studies provided some empirical support about the applicability of dual-process theories to explain and modify gamblingrelated erroneous cognition and gambling behavior, in some extent. More in detail, the intervention we developed following the dual-process model resulted was effective in reducing gambling-related erroneous cognitions in the short-term and in producing a stable change of these cognitions in the longterm. Additionally, there was a transfer of learning about gambling-related cognitions onto gambling behavior resulting in a reduction of gambling frequency after six months by the end of the intervention. Nonetheless, the medium effect size of this difference confirms the existing difficulties in changing gambling behavior among adolescents through educational interventions (Keen et al., 2016).

Concerning this point, the effectiveness of this intervention in reducing an important risk factor for maladaptive gambling behavior (i.e., gambling-erroneous cognitions) is very important as it has been showed that preventive programs that obtain change in risk and protective factors are more successful than programs showing behavior change (Foxcroft and Tsertsvadze, 2012). Moreover, these kind of programs focused on changing specific correlates of maladaptive gambling behavior may have effects that extend to other health behaviors (Hawkins et al., 2015).

Whereas this work has a number of strengths, including the evaluation of a theoretical model then linked to a gambling preventive intervention, and the use of good psychometric instruments, there are some limitations to take into account. First, as our work was conducted with boys attending Italian public high school, caution has to be paid about the generalizability of the present results. Future studies should be conducted in order to test the adequacy of the theoretical model with broader samples of adolescents, for instance including also girls. Moreover, although the descriptive data about gambling behavior were in line with previous data with adolescent males (e.g., Gupta et al., 2004; Olason et al., 2006), the small number of participants in Study 2 limits the impact of the current 
results concerning the developed preventive intervention. Future studies should be conducted with wider samples in order to evaluate the effectiveness of the intervention based on gambling behavior and severity. Finally, as research indicates that various and different factors increase the likelihood of problem gambling in adolescents (e.g., Donati et al., 2013; Cosenza and Nigro, 2015), it should be important to investigate theoretical models taking into account other variables in addition to susceptibility to the gambler's fallacy and superstitious thinking as predictors of gambling-erroneous cognitions.

\section{ETHICS STATEMENT}

This study was carried out in accordance with the APA recommendations of psychology deontology with written informed consent from all subjects. All subjects gave written informed consent in accordance with the Declaration of Helsinki. The protocol was approved by the ethical committees of each school.

\section{REFERENCES}

American Psychiatric Association (1987). Diagnostic and Statistical Manual of Mental Disorders, 3rd Edn. Washington, DC: American Psychiatric Association.

Arcan, K., and Karanci, A. N. (2015). Adaptation study of the Turkish version of the gambling-related cognitions scale (GRCS-T). J. Gambl. Stud. 31, 211-224. doi: 10.1007/s10899-013-9414-5

Bentler, P. M. (1990). Comparative fit indexes in structural models. Psychol. Bull. 107, 238-246. doi: 10.1037/0033-2909.107.2.238

Blinn-Pike, L., Worthy, S. L., and Jonkman, J. N. (2010). Adolescent gambling: a review of an emerging field of research. J. Adolesc. Health 47, 223-236. doi: 10.1016/j.jadohealth.2010.05.003

Calado, F., Alexandre, J., and Griffiths, M. D. (2016). Prevalence of adolescent problem gambling: a systematic review of recent research. J. Gambl. Stud. 33, 397-424. doi: 10.1007/s10899-016-9627-5

Capitanucci, D., Smaniotto, R., and Biganzoli, A. (2010). La prevenzione del gioco d'azzardo problematico negli adolescenti attraverso l'adattamento del video Lucky. Quad. Ital. Psichiatr. 29, 30-39. doi: 10.1016/j.quip.2010. 04.002

Chiesi, F., Donati, M. A., Galli, S., and Primi, C. (2013). The suitability of the SOGS-RA as screening tool: item response theory-based evidence. Psychol. Addict. Behav. 27, 287-293. doi: 10.1037/a0029987

Chiesi, F., Donati, M. A., Papi, C., and Primi, C. (2010). Misurare il pensiero superstizioso nei bambini: validità e attendibilità della Superstitious Thinking Scale [Measuring superstitious thinking in children: validity and reliability of the Superstitious Thinking Scale]. Età Evol. 97, 9-19.

Chiu, J., and Storm, L. (2010). Personality, perceived luck and gambling attitudes as predictors of gambling involvement. J. Gambl. Stud. 26, 205-227. doi: 10.1007/ s10899-009-9160-x

Clark, L. (2010). Decision-making during gambling: an integration of cognitive and psychobiological approaches. Philos. Trans. R. Soc. 365, 319-330. doi: 10.1098/ rstb.2009.0147

Colasante, E., Gori, M., Bastiani, L., Scalese, M., Siciliano, V., and Molinaro, S. (2014). Italian adolescent gambling behaviour: psychometric evaluation of the South Oaks Gambling Screen-Revised for Adolescents (SOGS-RA) among a sample of Italian students. J. Gambl. Stud. 30, 789-801. doi: 10.1007/s10899013-9385-6

Cosenza, M., and Nigro, G. (2015). Wagering the future: cognitive distortions, impulsivity, delay discounting, and time perspective in adolescent gambling. J. Adolesc. 45, 56-66. doi: 10.1016/j.adolescence.2015. 08.015

\section{AUTHOR CONTRIBUTIONS}

MD developed the theoretical model and constructed the intervention, which she conducted in the school setting. FC collaborated in the data analysis and revised the preliminary versions of the paper. AI promoted the research project among the schools and contacted the school headmasters. AM and FF participated in data interpretation. CP supervised the entire research project and gave her contribution in the theoretical and practical discussion of the results.

\section{ACKNOWLEDGMENTS}

The authors wish to thank all the students that have taken part in this research project, their school principal and teachers, as well as the undergraduate students for their collaboration in collecting data. They also wish to thank Rosanna Scopetani, Alessandro Orsetti, Ginetta Fusi, and Debora Vené for their participation in implementing the intervention in the school setting.

Delfabbro, P., Lambos, C., King, D., and Puglies, S. (2009). Knowledge and beliefs about gambling in Australian secondary school students and their implications for education strategies. J. Gambl. Stud. 25, 523-539. doi: 10.1007/s10899-0099141-0

Derevensky, J. L., Sklar, A., Gupta, R., and Messerlian, C. (2010). An empirical study examining the impact of gambling advertisements on adolescent gambling attitudes and behaviors. Int. J. Ment. Health Addict. 8, 21-34. doi: 10.1007/s11469-009-9211-7

Donati, M. A., Ancona, F., Chiesi, F., and Primi, C. (2015). Psychometric properties of the Gambling Related Cognitions Scale (GRCS) in young Italian gamblers. Addict. Behav. 45, 1-7. doi: 10.1016/j.addbeh.2015.01.001

Donati, M. A., Chiesi, F., and Primi, C. (2013). A model to explain at risk/problem gambling among male and female adolescents: gender similarities and differences. J. Adolesc. 36, 129-137. doi: 10.1016/j.adolescence.2012. 10.001

Donati, M. A., Primi, C., and Chiesi, F. (2014). Prevention of problematic gambling behavior among adolescents: testing the efficacy of an integrative intervention. J. Gambl. Stud. 30, 803-818. doi: 10.1007/s10899-0139398-1

Dowling, N. A. (2013). "The cognitive-behavioral treatment of female problem gambling," in The Wiley-Blackwell Handbook of Disordered Gambling, eds D. C. S. Richard, A. Blaszczynski, and L. Nower (Chichester: John Wiley \& Sons Ltd.).

Edgren, R., Castrén, S., Mäkelä, M., Pörtfors, P., Alho, H., and Salonen, A. H. (2016). Reliability of instruments measuring at-risk and problem gambling among young individuals: a systematic review covering years 2009-2015. J. Adolesc. Health 58, 600-615. doi: 10.1016/j.jadohealth.2016. 03.007

Evans, J. S. B., and Stanovich, K. E. (2013). Dual-process theories of higher cognition: advancing the debate. Perspect. Psychol. Sci. 8, 223-241. doi: 10.1177/ 1745691612460685

Ferland, F., Ladoucer, R., and Vitaro, F. (2002). Prevention of problem gambling: modifying misconceptions and increasing knowledge. J. Gambl. Stud. 18, 19-29. doi: 10.1023/A:1014528128578

Ferland, F., Ladouceur, R., and Vitaro, F. (2005). Efficiency of a gambling prevention program for youths: results from the pilot study. Encephale 31, 427-436. doi: 10.1016/S0013-7006(05)82404-2

Flay, B. R., Biglan, A., Boruch, R. F., Castro, F. G., Gottfredson, D., Kellam, S., et al. (2005). Standards of evidence: criteria for efficacy, effectiveness and dissemination. Prev. Sci. 6, 151-175. doi: 10.1007/s11121-0055553-y 
Fortune, E. E., and Goodie, A. S. (2012). Cognitive distortions as a component and treatment focus of pathological gambling: a review. Psychol. Addict. Behav. 26, 298-310. doi: 10.1037/a0026422

Foxcroft, D. R., and Tsertsvadze, A. (2012). Universal alcohol misuse prevention programmes for children and adolescents: Cochrane systematic reviews. Perspect. Public Health 132, 128-134. doi: 10.1177/1757913912 443487

Gaboury, A., and Ladouceur, R. (1993). Evaluation of a prevention program for pathological gambling among adolescents. J. Prim. Prev. 14, 21-28. doi: 10. 1007/BF01324653

Gibbons, F. X., Houlihan, A. E., and Gerrard, M. (2009). Reason and reaction: the utility of a dual-focus, dual-processing perspective on promotion and prevention of adolescent health risk behaviour. Br. J. Health Psychol. 14, 231-248. doi: 10.1348/135910708X376640

Gilovich, T., Griffin, D., and Kahneman, D. (2002). Heuristics and Biases: The Psychology of Intuitive Judgment. Cambridge: Cambridge University Press. doi: 10.1017/CBO9780511808098

Goodie, A., and Fortune, E. (2013). Measuring cognitive distortions in pathological gambling: review and meta-analyses. Psychol. Addict. Behav. 27, 730-743. doi: 10.1037/a0031892

Granero, R., Penelo, E., Stinchfield, R., Fernandez-Aranda, F., Savvidou, L. G., Fröberg, F., et al. (2014). Is pathological gambling moderated by age? J. Gambl. Stud. 30, 475-492. doi: 10.1007/s10899-0139369-6

Gupta, R., Derevensky, J., and Marget, N. (2004). Coping strategies employed by adolescents with gambling problems. Child Adolesc. Ment. Health 9, 115-120. doi: 10.1111/j.1475-3588.2004.00092.x

Hawkins, J. D., Jenson, J. M., Catalano, R., Fraser, M. W., Botvin, G. J., Shapiro, V., et al. (2015). Unleashing the Power of Prevention. Washington, DC: National Research Council.

Huic, A., Kranzelic, V., Hundric, D. D., and Ricijas, N. (2017). Who really wins? Efficacy of a Croatian youth gambling prevention program. J. Gambl. Stud. 33, 1011-1033. doi: 10.1007/s10899-017-9668-4

Iliceto, P., Fino, E., Cammarota, C., Giovani, E., Petrucci, F., Desimoni, M., et al. (2015). Factor structure and psychometric properties of the Italian version of the Gambling Related Cognition Scale (GRCS-I). J. Gambl. Stud. 31, 225-242. doi: 10.1007/s10899-013-9405-6

Johansson, A., Grant, J. E., Kim, S. W., Odlaug, B. L., and Götestam, K. G. (2009). Risk factors for problematic gambling: a critical literature review. J. Gambl. Stud. 25, 67-92. doi: 10.1007/s10899-008-9088-6

Kahneman, D., Slovic, P., and Tversky, A. (1982). Judgments under Uncertainty. Cambridge: Heuristics and Biases. doi: 10.1017/CBO978051180 9477

Keen, B., Blaszczynski, A., and Anjoul, F. (2016). Systematic review of empirically evaluated school-based gambling education programs. J. Gambl. Stud. 33, 301-325. doi: 10.1007/s10899-016-9641-7

Klaczynski, P. A. (2004). "A dual-process model of adolescent development: implications for decision making, reasoning, and identity," in Advances in Child Development and Behavior, ed. R. V. Kail (San Diego, CA: Academic Press), $73-123$.

Kokis, J. V., MacPherson, R., Toplak, M. E., West, R. F., and Stanovich, K. E. (2002). Heuristic and analytic processing: age trends and associations with cognitive ability and cognitive styles. J. Exp. Child Psychol. 83, 26-52. doi: 10.1016/S00220965

Ladouceur, R., Goulet, A., and Vitaro, F. (2013). Prevention programmes for youth gambling: a review of the empirical evidence. Int. Gambl. Stud. 13, 141-159. doi: 10.1080/14459795.2012.740496

Ninness, H. A. C., and Ninness, S. K. (1998). Superstitious math performance: interactions between rule-governed and scheduled contingencies. Psychol. Rec. 48, 45-62. doi: 10.1007/BF03395258

Olason, D. T., Sigurdardottir, K. J., and Smari, J. (2006). Prevalence estimates of gambling participation and problem gambling among 16-18-year-old students in Iceland: a comparison of the SOGS-RA and DSM-IV-MR-J. J. Gambl. Stud. 22, 23-39. doi: 10.1007/s10899-005-9001-5

Perkins, D. N. (1995). Outsmarting IQ: The Emerging Science of Learnable Intelligence. New York, NY: Free Press.

Primi, C., and Chiesi, F. (2011). "The role of relevant knowledge and cognitive ability in gambler fallacy," in Proceedings of the 7th Congress of the European Society for Research in Mathematics Education (CERME), Rzeszow.

Raylu, N., and Oei, T. P. (2004). The gambling related cognitions scale (GRCS): development, confirmatory factor validation and psychometric properties. Addiction 99, 757-769. doi: 10.1111/j.1360-0443.2004. 00753.x

Ricijas, N., Hundric, D. D., and Huic, A. (2016). Predictors of adverse gambling related consequences among adolescent boys. Child. Youth Serv. Rev. 67, 168-176. doi: 10.1016/j.childyouth.2016.06.008

Sá, W. C., Kelley, C. N., Ho, C., and Stanovich, K. E. (2005). Thinking about personal theories: individual differences in the coordination of theory and evidence. Pers. Individ. Dif. 38, 1149-1161. doi: 10.1016/j.paid.2004. 07.012

Skoukaskas, N., and Satkeviciute, R. (2007). Adolescent pathological gambling in Kaunas, Lithuania. Nord. J. Psychiatry 61, 86-91. doi: 10.1080/08039480701226054

Splevins, K., Mireskandari, S., Clayton, K., and Blaszczynski, A. (2010). Prevalence of adolescent problem gambling, related harms and help-seeking behaviours among an Australian population. J. Gambl. Stud. 26, 189-204. doi: 10.1007/ s10899-009-9169-1

Stanovich, K. E. (2004). The Robot's Rebellion: Finding Meaning in the Age of Darwin. Chicago, IL: University of Chicago Press. doi: 10.7208/chicago/ 9780226771199.001.0001

Steiger, J. H., and Lind, J. C. (1980). "Statistically based tests for the number of common factors," in Proceedings of the Annual Meeting of the Psychometric Society, Vol. 758, Iowa City, IA, 424-453.

St-Pierre, R. A., Temcheff, C. E., Derevensky, J. L., and Gupta, R. (2015). Theory of planned behavior in school-based adolescent problem gambling prevention: a conceptual framework. J. Prim. Prev. 36, 361-385. doi: 10.1007/s10935-0150404-5

Taylor, R. N., Parker, J. D., Keefer, K. V., Kloosterman, P. H., and Summerfeldt, L. J. (2014). Are gambling related cognitions in adolescence multidimensional?: factor structure of the gambling related cognitions scale. J. Gambl. Stud. 30, 453-465. doi: 10.1007/s10899-013-9368-7

Toneatto, T. (1999). Cognitive psychopathology of problem gambling. Subst. Use Misuse 34, 1593-1604. doi: 10.3109/1082608990903 9417

Toplak, M., Liu, E., Macpherson, R., Toneatto, T., and Stanovich, K. E. (2007). The reasoning skills and thinking dispositions of problem gamblers: a dual-process taxonomy. J. Behav. Decis. Mak. 20, 103-124. doi: 10.1002/ bdm. 544

Tucker, L. R., and Lewis, C. (1973). A reliability coefficient for maximum likelihood factor analysis. Psychometrika 38, 1-10. doi: 10.1007/BF02291170

Turner, N. E., Macdonald, J., Bartoshuk, M., and Zangeneh, M. (2008). The evaluation of a 1 -h prevention program for problem gambling. Int. J. Ment. Health Addict. 6, 238-243. doi: 10.1007/s11469-007-9121-5

Tversky, A., and Kahneman, D. (1971). Belief in the law of small numbers. Psychol. Bull. 76:105. doi: 10.1037/h0031322

Vitaro, F., Wanner, B., Ladouceur, R., Brendgen, M., and Tremblay, R. E. (2004). Trajectories of gambling during adolescence. J. Gambl. Stud. 20, 47-69. doi: 10.1023/B:JOGS.0000016703.84727.d3

Volberg, R. A., Gupta, R., Griffiths, M. D., Ólason, D. T., and Delfabbro, P. (2010). An international perspective on youth gambling prevalence studies. Int. J. Adolesc. Med. Health 22, 3-38.

Welte, J. W., Barnes, G. M., Tidwell, M. C. O., and Hoffman, J. H. (2009). The association of form of gambling with problem gambling among American youth. Psychol. Addict. Behav. 23, 105-112. doi: 10.1037/ a0013536

West, R. F., Toplak, M. E., and Stanovich, K. E. (2008). Heuristics and biases as measures of critical thinking: associations with cognitive ability and thinking dispositions. J. Educ. Psychol. 100, 930-941. doi: 10.1037/ a0012842

Wickwire, E. M., Whelan, J. P., and Meyers, A. M. (2010). Outcome expectancies and gambling behavior among urban adolescents. Psychol. Addict. Behav. 24, 75-88. doi: 10.1037/a0017505

Wickwire, E. M., Whelan, J. P., Meyers, A. W., and Murray, D. M. (2007). Environmental correlates of gambling behavior in urban adolescents. J. Abnorm. Psychol. 35, 179-190. doi: 10.1007/s10802-006-9065-4 
Williams, R. (2002). Prevention of Problem Gambling: A School-based Intervention: Final Report. Calgary, AB: Alberta Gaming Research Institute.

Williams, R. J., Wood, R. T., and Currie, S. R. (2010). Stacked deck: an effective, school-based program for the prevention of problem gambling. J. Prim. Prev. 31, 109-125. doi: 10.1007/s10935-010-0212-x

Winters, K. C., Stinchfield, R., and Kim, L. (1995). Monitoring adolescent gambling in Minnesota. J. Gambl. Stud. 11, 165-183. doi: 10.1007/BF02 107113

Winters, K. C., Stinchfield, R. D., and Fulkerson, J. (1993). Toward the development of an adolescent gambling problem severity scale. J. Gambl. Stud. 9, 63-84. doi: 10.1007/BF01019925
Conflict of Interest Statement: The authors declare that the research was conducted in the absence of any commercial or financial relationships that could be construed as a potential conflict of interest.

Copyright (c) 2018 Donati, Chiesi, Iozzi, Manfredi, Fagni and Primi. This is an open-access article distributed under the terms of the Creative Commons Attribution License (CC BY). The use, distribution or reproduction in other forums is permitted, provided the original author(s) or licensor are credited and that the original publication in this journal is cited, in accordance with accepted academic practice. No use, distribution or reproduction is permitted which does not comply with these terms. 